\title{
Leprosy in Portugal 1946-80: epidemiologic patterns observed during declining incidence rates
}

\author{
L M IRGENS*, F MELO CAEIRO + \& M F LECHAT $\ddagger$ \\ *Department of Hygiene and Social Medicine, University of Bergen, \\ Armauer Hansen Bldg. MFH, Haukeland Hospital, N-5021 Bergen, \\ Norway; †Direccao Geral dos Cuidados de Saude Primarios, \\ Alameda D. Afonso Henriques, 45, P 1056 Lisbon Codex, Portugal; \\ $\ddagger$ Department of Epidemiology and Preventive Medicine, Catholic \\ University of Louvain, Clos Chapelle aux Champs 30, B 1200 \\ Brussels, Belgium
}

Accepted for publication 10 August 1989

\begin{abstract}
Summary Compulsory notification of leprosy in Portugal formed the basis for the establishment of a national patient registry used in an epidemiological study. Highest incidence rates were observed in the coastal counties in the middle of Portugal and particularly in the municipalities with a high annual rainfall. Peak incidence rate in males was observed at the age of 25-29 years against 50-59 in females. A continuous and increasing decline in incidence rates was observed throughout the observation period, 1946-80. Towards the end of the period the slopes of the incidence curves seemed to be identical with those observed in other countries where leprosy has previously been eradicated. This is consistent with the notion that towards the end of an endemic situation no new transmission of the disease occurs, and the incidence curve takes the shape of the right part of the distribution of incubation periods which apparently is uniform in leprosy, irrespective of time and place. The pattern observed in other areas during declining incidence rates, of an increase in age at onset by year of onset together with a lack of increase in age at onset by year of birth was confirmed by the Portuguese data, also consistent with a break in the transmission of the disease a long time before the final termination of the endemic situation.
\end{abstract}

\section{Introduction}

Data necessary to assess secular trends in leprosy have been scarce. Apart from the National Leprosy Registry of Norway, ${ }^{1}$ national schemes for the provision of data necessary for community based planning, administration, and evaluation of leprosy control programmes on the one hand and for epidemiological studies of leprosy on the other have been rare. However, during the last decades, the importance of careful registration of leprosy patients has been realized and stressed in an increasing number of 
countries. Thus, sources of data (e.g. Refs 2-9) have eventually evolved that are useful in epidemiological studies.

In Portugal, the necessity of acquiring data for planning, administration and evaluation of leprosy control programmes was realized rather early. ${ }^{2}$ Thus, in 1947 , the notification of all leprosy cases to a central agency was legally authorized and over the years a considerable amount of data has been filed.

Today, there is a need in many countries to evaluate leprosy control programmes and to assess secular trends. In Portugal, opportunity is offered to meet this need.

Based on some of the information at hand, a computerized register has been established, comprising all cases of leprosy in the country notified after 1945. This register provided the source material for the present analyses which attempt to elucidate the epidemiology of leprosy and to obtain knowledge relevant to control programmes in areas where leprosy is still prevalent.

\section{Materials and methods}

NOTIFICATION AND REGISTRATION OF LEPROSY CASES

Compulsory notification of leprosy cases was introduced by a decree of 2 August 1947. The central registry was established at the National Centre for Leprosy, Hospital Rovisco Pais (the Centre), in the middle of the country. Concomitantly, mandatory hospitalization was introduced for infectious patients, unless satisfactory domiciliary care could be provided. Activities under this decree were supervised by Instituto de Assistencia aos Leprosos (the Institute) affiliated with the General Directorate of Health in Lisbon. Diagnostic criteria were established by the Centre for the definition of leprosy and for the various types of the disease. The Madrid classification was adopted in 1953 and replaced by the Ridley-Jopling classification in 1968-69. Close contacts were established with the faculties training the medical profession.

Every physician in the country was required to notify all the leprosy cases he diagnosed. In the case finding and reporting the physicians were assisted by a number of mobile teams established by the Centre in 1958. Each team consisted of a physician, a nurse and a social worker, who were responsible for patients under domiciliary care. The mobile teams played an important role in the standardization of the reporting with respect to diagnostic criteria and regimens for treatment and follow-up. After 1973, their functions were gradually transferred to the general health centres, or to specialized outpatients' departments in the larger cities.

An individual notification form (Table 1) was filled out for each patient, by the physician, and sent to the Centre. After 1976 a copy of the form was sent to the Institute as well. When changes were observed in the condition of the patient the central agencies were notified.

By a decree of 1976 the Institute was made responsible for the organization and administration of all leprosy control activities. Accordingly a central file consisting of all notified cases was established for epidemiological and administrative purposes. At the same time, mandatory hospitalization of infectious cases was abolished. Gradually the activities became more decentralized, and in 1984 a third decree actually closed the Institute. Its objectives, however, were maintained and integrated together with all 
Table 1. Items of personal data used in the notification of leprosy cases in Portugal

Notifying unit
Name
Central serial number
Date of registration
Year of onset
Date of birth
Birthplace
Place of residence at onset
Place of residence at registration
Occupation
Education
Sex
Marital status

\author{
Clinical data: \\ History including reactions \\ Symptoms and signs \\ Lepromine reaction \\ Tuberculin reaction \\ Microbiological findings \\ Histological findings \\ Type of leprosy \\ Other diseases
}

\section{Relatives:}

Names and relationships with lepromin reaction and central serial number (if affected with leprosy)

Follow up information:

Subsequent microbiological findings

Year when cured

Year of death

organizational structures into the division of Primary Health Care under The Ministry of Health.

\section{ADDITIONAL SOURCES OF DATA}

Data on the total population were derived from general population censuses conducted in 1940, 1950, 1960, 1970 and 1981, published by the National Institute of Statistics. Information on emigrants and their eventual return to Portugal was obtained from official statistics, and meteorological observations obtained during 1931-60 on precipitation, air humidity and temperature were provided by the National Meteorological Services. ${ }^{3}$

\section{METHODS}

From 1980 onwards data were transferred to a computerized register. As the basis for computer registration, a photocopy was used of the notification form, after the necessary codes were added. Items of personal data are shown in Table 2. Identification of the forms was secured during the computer registration by the consecutive generation of identification numbers. Furthermore, each patient, when first registered, had been allotted a central serial number generated by the Institute identifying all patient notifications.

Registration errors were to a large extent controlled by registering all forms twice for computerized comparison. Inequalities were detected and corrected. To avoid the registration of unacceptable values or invalid codes, valid ranges and codes were specified for each item of personal data. Inevitably, some patients, for various reasons, had more than one notification form. To avoid duplicate records the forms were marked with the central serial number of the patients.

Case ascertainment could not be directly assessed because no independent source of information was available. Yet the local system of case finding, enforced since 1947 with 
Table 2. Items of personal data transferred to the computer file

\begin{tabular}{ll} 
Identification no. (generated) & Type of leprosy \\
Central serial no. & Lepromin reaction \\
$\begin{array}{l}\text { Date of registration } \\
\text { Year of onset }\end{array}$ & Year of cure \\
$\begin{array}{l}\text { Date of birth } \\
\text { Birthplace }\end{array}$ & Year of death \\
Place of residence at onset & Relatives: \\
Place of residence at registration & for each: \\
$\begin{array}{l}\text { Occupation } \\
\text { Sex }\end{array}$ & Central serial no. (if ill) \\
Marital status & Relationship \\
& Lepromin reaction \\
& Ill-not ill. \\
\hline
\end{tabular}

compulsory notification entrusted to all physicians, assisted by the mobile teams supervising and guiding health personnel at the local level, should provide sufficient ascertainment. In addition, case ascertainment was indirectly evaluated on the basis of register delay, i.e. the period between onset and registration (Table 3). A long delay might cause under-registration. Obviously, this delay decreased during the first years after 1947. Measured as median difference between years of registration and onset, the delay remained below $1 \cdot 3$ years after 1950 .

Likewise, over-registration, i.e. cases being falsely diagnosed as leprosy, could not be directly assessed due to lack of sources of information. Forms representing such cases were immediately removed from the files by the central agencies. However, the careful follow-up examinations, organized and conducted by the mobile teams, ensures that the number of such cases not removed from the files has remained low.

Completeness of reporting in terms of stated year and place of birth, year of onset, place of residence, and type of leprosy increased from 1947 onwards (Table 4).

The study period was defined to cover 1946-80. In spite of an increasing case

Table 3. New cases of leprosy in Portugal with median of the delay period between onset and registration, by year of onset

\begin{tabular}{lcc}
\hline Year of onset & $\begin{array}{c}\text { Number } \\
\text { of patients }\end{array}$ & $\begin{array}{c}\text { Delay } \\
\text { (median years) }\end{array}$ \\
\hline $1947-50$ & 615 & $1 \cdot 8$ \\
$1951-55$ & 647 & $1 \cdot 3$ \\
$1956-60$ & 512 & $0 \cdot 8$ \\
$1961-65$ & 344 & $1 \cdot 1$ \\
$1966-70$ & 239 & $0 \cdot 8$ \\
$1971-75$ & 168 & $1 \cdot 2$ \\
$1976-80$ & 104 & $1 \cdot 0$ \\
\hline $1947-80$ & 2629 & $1 \cdot 2$ \\
-1947 & 1194 & \\
$1981-83$ & 32 & \\
Unknown & 646 & \\
\hline Total & 4501 & \\
\hline
\end{tabular}


Table 4. Incompleteness in reporting of data on leprosy patients in Portugal, 1947-80, by year of registration

\begin{tabular}{lcccc}
\hline & \multicolumn{4}{c}{ Lack in registration of } \\
\cline { 2 - 5 } & $\begin{array}{c}\text { Year of } \\
\text { birth } \\
\text { Year of }\end{array}$ & $\begin{array}{c}\text { Birth } \\
\text { place } \\
\text { registration }\end{array}$ & $\begin{array}{c}\text { Year of } \\
\text { onset } \\
(\%)\end{array}$ & $\begin{array}{c}\text { Type of } \\
\text { leprosy } \\
(\%)\end{array}$ \\
\hline $1947-50$ & $14 \cdot 5$ & $0 \cdot 3$ & $13 \cdot 0$ & $5 \cdot 4$ \\
$1951-55$ & 12.9 & $0 \cdot 3$ & $6 \cdot 9$ & $2 \cdot 5$ \\
$1956-60$ & $11 \cdot 2$ & 0 & $7 \cdot 0$ & $0 \cdot 4$ \\
$1961-65$ & $8 \cdot 2$ & $0 \cdot 4$ & $6 \cdot 6$ & $0 \cdot 6$ \\
$1966-70$ & $3 \cdot 1$ & 0 & $3 \cdot 7$ & $0 \cdot 3$ \\
$1971-75$ & $2 \cdot 8$ & 0 & $12 \cdot 7$ & $1 \cdot 4$ \\
$1976-80$ & $3 \cdot 6$ & $1 \cdot 5$ & $11 \cdot 9$ & $1 \cdot 5$ \\
\hline Average & $8 \cdot 04$ & $0 \cdot 3$ & $8 \cdot 8$ & $1 \cdot 7$ \\
\hline
\end{tabular}

ascertainment during the first years after 1947, these years were included. To confine the epidemiological analyses only to the later decades, e.g. after 1950-55, would imply the loss of a considerable amount of important information. Furthermore, most analyses are based on year of onset rather than year of registration which to some extent adjust for the increased activities in case finding after 1947. Still, some patients taken ill in 1946 might have died before the scheme of notification was introduced in 1947. However, even the 5yearcase fatality rate in untreated leprosy in Norway in the last century was no more than $5 \%{ }^{1}$ which probably is an overestimate in the present situation. Accordingly, the inclusion of the year 1946 was considered justified.

The analyses were restricted to continental Portugal with a population in 1960 of approximately 8,890,000 inhabitants, increasing from 7,920,000 in 1940 to 9,290,000 in 1981. The municipality (concelho) was the smallest geographical unit for reporting the residence of the patients, aggregating into 18 districts (distrito).

When not otherwise specified, incidence rate in the present study refers to year of onset rather than year of registration. In each case the year was recorded when the first symptom was observed by the patient. Insidious onset and social stigma are factors which might influence the patient's recall, tending to postpone the time of onset. Still, year of onset is preferred as the basis for the present analyses relating more to epidemiologic aspects than the purely administrative implications of year of registration. Incidence rate is defined as annual number of new patients, according to year of onset, per 100,000 population.

Average incidence rate is an attack rate referring to the complete observation period and is defined as total number of patients whose year of onset was within the observation period, multiplied by 100,000 and divided by the number of inhabitants in the middle of the observation period, i.e. in 1960 and by the length of the observation period, i.e. 35 years.

Sex ratio was calculated as the ratio between male and female incidence rates multiplied by 100 .

Type index was calculated as the lepromatous (LL and LB) fraction of all patients. Clinical form was recorded as lepromatous, borderline, tuberculoid, indeterminate (or 
previous clinically equivalent diagnostic groups) or unknown and refers to the form reported at registration.

Possible statistical associations between incidence rates and a set of independent, environmental variables were tested in simple and stepwise linear regression analyses. ${ }^{12}$

\section{Results}

Of 4501 patients registered, 1659 were considered to have had their onset prior to 1946 and 34 after 1980. Furthermore, place of residence at onset was unknown in 6 cases. Thus 2802 cases occurring during the observation period remained for analysis.

\section{GEOGRAPHICAL DISTRIBUTION}

The overall average incidence rate amounted to 0.86 per 100,000 per year, varying, however, considerably from district to district (Figure 1, Table 5). Apart from Faro (2.06)

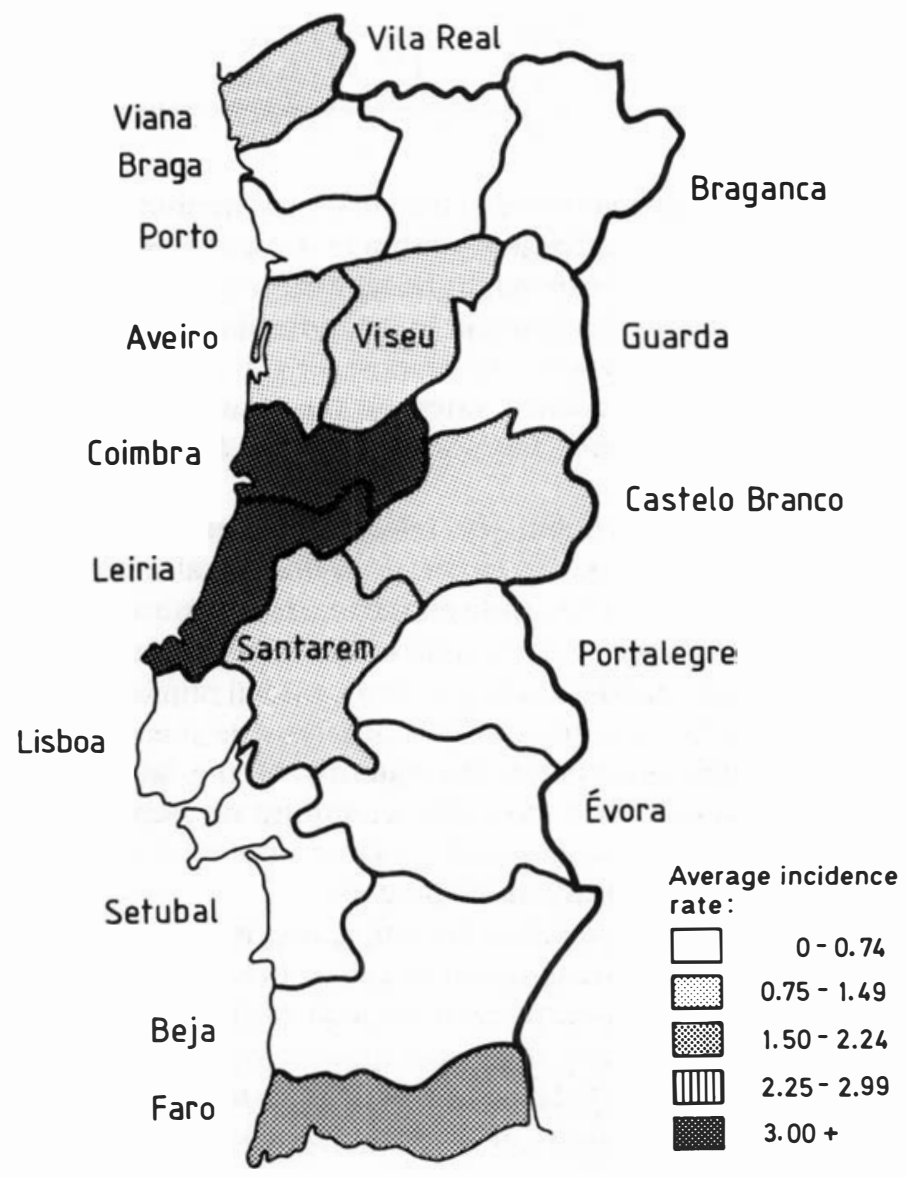

Figure 1. Average incidence rates of leprosy in Portugal, 1946-80 by district. 
Table 5. Incidence rates of leprosy in continental Portugal by year of onset and district of residence at onset

\begin{tabular}{|c|c|c|c|c|c|c|c|c|}
\hline \multirow{2}{*}{$\begin{array}{l}\text { District of } \\
\text { residence }\end{array}$} & \multicolumn{8}{|c|}{ Year of onset } \\
\hline & $1941-45$ & $1946-50$ & $1951-55$ & $1956-60$ & $1961-65$ & $1966-70$ & $1971-75$ & $1976-80$ \\
\hline Aveiro & $1 \cdot 62$ & $2 \cdot 98$ & $1 \cdot 66$ & $1 \cdot 40$ & $1 \cdot 06$ & $0 \cdot 59$ & $0 \cdot 46$ & $0 \cdot 20$ \\
\hline Beja & 0.07 & $0 \cdot 14$ & $0 \cdot 28$ & - & $0 \cdot 54$ & 0.45 & $0 \cdot 70$ & - \\
\hline Braga & $0 \cdot 84$ & $1 \cdot 54$ & 0.93 & $0 \cdot 86$ & $0 \cdot 37$ & 0.49 & $0 \cdot 22$ & $0 \cdot 15$ \\
\hline Braganca & 0.09 & 0.09 & $0 \cdot 26$ & $0 \cdot 17$ & $0 \cdot 55$ & $0 \cdot 10$ & - & - \\
\hline Castelo-Branco & $1 \cdot 29$ & $2 \cdot 32$ & $2 \cdot 11$ & $1 \cdot 45$ & $0 \cdot 73$ & 0.44 & $0 \cdot 24$ & $0 \cdot 17$ \\
\hline Coimbra & $3 \cdot 18$ & $6 \cdot 71$ & $6 \cdot 19$ & $4 \cdot 01$ & $2 \cdot 77$ & $2 \cdot 54$ & $1 \cdot 56$ & $0 \cdot 61$ \\
\hline Evora & $0 \cdot 19$ & - & $0 \cdot 18$ & - & $0 \cdot 10$ & - & - & - \\
\hline Faro & $2 \cdot 18$ & 3.99 & $3 \cdot 21$ & $2 \cdot 33$ & $2 \cdot 11$ & $1 \cdot 14$ & $0 \cdot 85$ & $0 \cdot 84$ \\
\hline Guarda & $0 \cdot 27$ & $0 \cdot 52$ & $0 \cdot 27$ & $0 \cdot 07$ & $0 \cdot 07$ & $0 \cdot 35$ & $0 \cdot 19$ & $0 \cdot 19$ \\
\hline Leiria & $4 \cdot 80$ & $7 \cdot 05$ & $6 \cdot 44$ & $5 \cdot 32$ & $2 \cdot 61$ & 0.99 & $0 \cdot 67$ & $0 \cdot 29$ \\
\hline Lisboa & $0 \cdot 23$ & $0 \cdot 46$ & $0 \cdot 38$ & $0 \cdot 34$ & $0 \cdot 32$ & $0 \cdot 12$ & $0 \cdot 16$ & $0 \cdot 15$ \\
\hline Portalegre & 0.42 & $0 \cdot 20$ & $0 \cdot 61$ & $0 \cdot 21$ & $0 \cdot 34$ & $0 \cdot 38$ & $0 \cdot 28$ & $0 \cdot 28$ \\
\hline Porto & 0.53 & $0 \cdot 88$ & 0.61 & 0.55 & $0 \cdot 33$ & $0 \cdot 36$ & $0 \cdot 19$ & $0 \cdot 11$ \\
\hline Santarem & $1 \cdot 48$ & $2 \cdot 22$ & 1.65 & $1 \cdot 21$ & $0 \cdot 79$ & $0 \cdot 50$ & $0 \cdot 37$ & $0 \cdot 23$ \\
\hline Setubal & $0 \cdot 89$ & 1.99 & $1 \cdot 24$ & 0.66 & $0 \cdot 50$ & $0 \cdot 40$ & $0 \cdot 16$ & 0.07 \\
\hline Viana do Castelo & $1 \cdot 74$ & $2 \cdot 11$ & $2 \cdot 52$ & $2 \cdot 45$ & $1 \cdot 33$ & 0.93 & $0 \cdot 40$ & $0 \cdot 16$ \\
\hline Vila Real & $1 \cdot 01$ & 0.64 & 0.93 & $1 \cdot 18$ & $1 \cdot 03$ & $0 \cdot 57$ & $0 \cdot 38$ & $0 \cdot 30$ \\
\hline Viseu & $1 \cdot 31$ & 1.93 & $1 \cdot 87$ & $1 \cdot 77$ & $1 \cdot 12$ & $0 \cdot 79$ & 0.44 & $0 \cdot 24$ \\
\hline
\end{tabular}

in the South, the disease was concentrated in the middle of the country and particularly in the two districts along the Atlantic Coast, Coimbra (3.45) and Leiria (3.24), rendering the impression that proximity to the ocean might be related to high incidence rates; however this association seemed to be relatively weak. In the municipalities bordering the Atlantic Ocean, the average incidence rate was 1.06 against 0.84 in the inland ones. In a simple linear regression of average incidence rates in the municipalities on whether the municipality was coastal or inland, the association was found to be not significant $(p>0 \cdot 05)$.

The geographical distribution might also relate to climatic variables. No association was found with mean air temperature. ${ }^{3}$ However in municipalities with a mean annual rainfall ${ }^{3}$ of $800 \mathrm{~mm}$ or more (Figure 2), the average incidence rate was 1.26 against 0.50 in the other municipalities implying a relative risk of $2 \cdot 5$. In a simple regression analysis of average incidence rates in the municipalities on mean annual rainfall, a strong association was found $(p=0.001)$. In a stepwise regression analysis of average incidence rates on mean annual rainfall and coast/inland, the entering of the latter variable increased multiple $\mathrm{R}$ significantly ( $p=0.0003$ ), implying an interaction between the two independent variables, i.e. among municipalities with the same amount of annual rainfall, highest incidence rates were found in coastal municipalities.

The organization of the municipalities encompassing in most cases urban as well as rural areas, precluded direct differentiation into urban and rural specific incidence rates. However, considering the main municipality in each district as more urban than the rest of the municipalities, average incidence rates of respectively 0.50 and 1.07 were observed, implying higher rates in rural dwellings. Even more pure urban areas were obtained by studying the rate in main municipalities of 100,000 inhabitants or more as recorded in the 1960 census. In these larger municipalities which all represent cities, an average incidence rate of 0.29 was observed. 


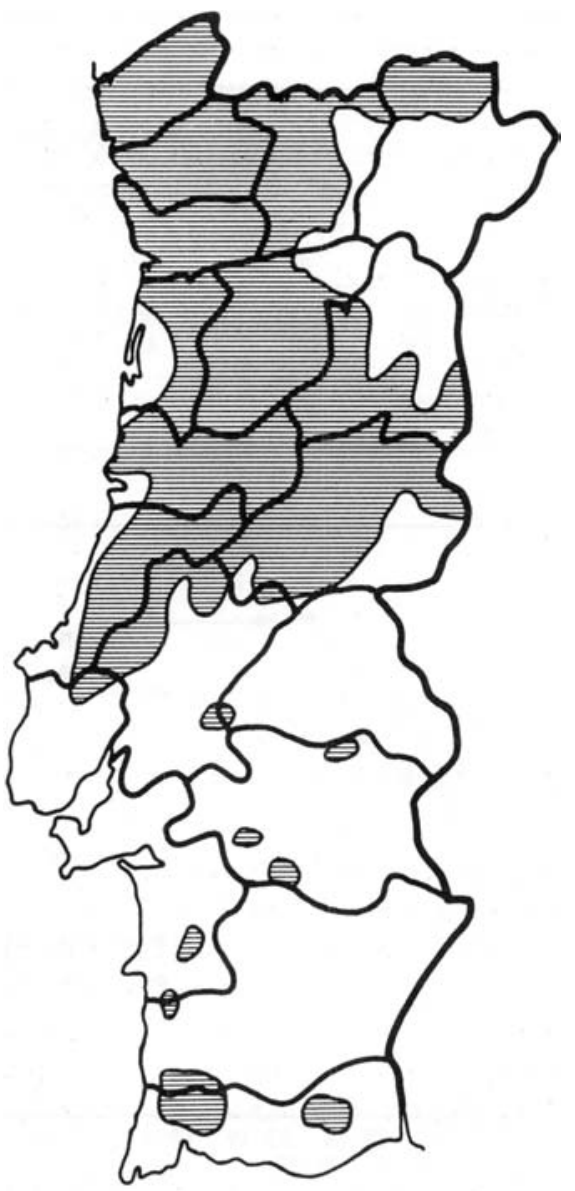

Figure 2. Areas in Portugal with a mean annual rainfall of $800 \mathrm{~mm}$ or more.

During the observation period population growth varied considerably from area to area. However, in a simple linear regression analysis of average incidence rate on relative population growth (increase 1930-60/population 1930), no association was found $(p>0 \cdot 05)$.

\section{DISTRIBUTION BY SEX, AGE AND TYPE}

Leprosy occurred more frequently in males than in females, the male proportion of all patients was $54 \cdot 6 \%$ (Table 6 ). Median age at onset was $34 \cdot 4$ years in males and $35 \cdot 1$ years in females. However, the distribution of cases by age at onset differed considerably; highest age specific incidence rate in males was observed at the age of 25-29 years while the highest rates in females was observed at the age of 50-59 years (Figure 3). Lepromatous leprosy represented the most common type of the disease with a type index of $61 \cdot 2 \% ; 63 \cdot 1 \%$ in males and $58.9 \%$ in females (Table 7 ). The age distribution varied 
Table 6. Leprosy cases in continental Portugal with year of onset, 1946-80, by sex and average incidence rate in the district of residence at onset

\begin{tabular}{|c|c|c|c|c|c|c|c|c|}
\hline \multirow[b]{3}{*}{ Sex } & \multirow[b]{3}{*}{ Total no. } & \multirow[b]{3}{*}{$\%$} & \multicolumn{6}{|c|}{ Average incidence rate in district } \\
\hline & & & \multicolumn{2}{|c|}{-0.74} & \multicolumn{2}{|c|}{$0 \cdot 75-3 \cdot 0$} & \multicolumn{2}{|c|}{$3 \cdot 1+$} \\
\hline & & & No. & $\%$ & No. & $\%$ & No. & $\%$ \\
\hline Male & 1529 & $54 \cdot 6$ & 467 & $59 \cdot 3$ & 568 & $54 \cdot 9$ & 494 & $50 \cdot 4$ \\
\hline Female & 1273 & $45 \cdot 4$ & 320 & $40 \cdot 7$ & 466 & $45 \cdot 1$ & 487 & $49 \cdot 6$ \\
\hline Total & 2802 & $100 \cdot 0$ & 787 & $100 \cdot 0$ & 1034 & $100 \cdot 0$ & 981 & $100 \cdot 0$ \\
\hline
\end{tabular}

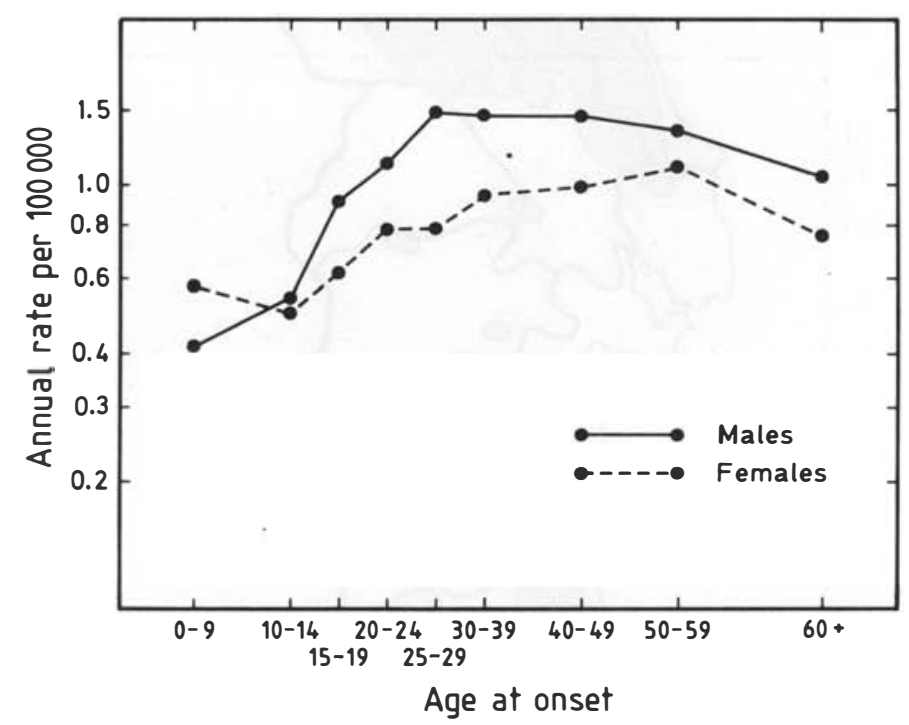

Figure 3. Age and sex-specific incidence rates of leprosy in continental Portugal, 1946-80.

according to the type of disease, with a high age at onset in tuberculoid cases, and particularly among females (Figure 4).

In these patterns geographical differences were observed. A relatively low proportion of male cases was particularly found in the high incidence districts (Leiria and Coimbra) (Table 6). In these districts a high proportion of patients was observed with onset before the age of $20(19 \cdot 6 \%)$ compared with the low incidence districts $(14 \cdot 3 \%)$ (Table 8$)$. Identical trends were found in males and females.

No association was found between the type index and average incidence rate of the district of residence, either in males or females (Table 7).

\section{SECULAR TRENDS}

In all parts of the country incidence rates declined continuously and with an increasing relative fall throughout the observation period (Figure 5(a)). The steepest fall was 
Table 7. Proportion of all leprosy cases with lepromatous leprosy by sex and average incidence rate in the district of residence of onset. Based on all cases in continental Portugal with year of onset $1946-80$

\begin{tabular}{lcccc}
\hline & & \multicolumn{3}{c}{ Average incidence rate in district } \\
\cline { 3 - 5 } Sex & $\begin{array}{c}\text { Total } \\
(\%)\end{array}$ & $\begin{array}{c}-0 \cdot 74 \\
(\%)\end{array}$ & $\begin{array}{c}0 \cdot 75-3 \cdot 0 \\
(\%)\end{array}$ & $\begin{array}{c}3 \cdot 1+ \\
(\%)\end{array}$ \\
\hline Male & $63 \cdot 1$ & $62 \cdot 5$ & $64 \cdot 1$ & $62 \cdot 6$ \\
Female & $58 \cdot 9$ & $57 \cdot 5$ & $62 \cdot 7$ & $56 \cdot 3$ \\
\hline Average & $60 \cdot 0$ & $60 \cdot 0$ & 63.4 & $59 \cdot 4$ \\
\hline
\end{tabular}

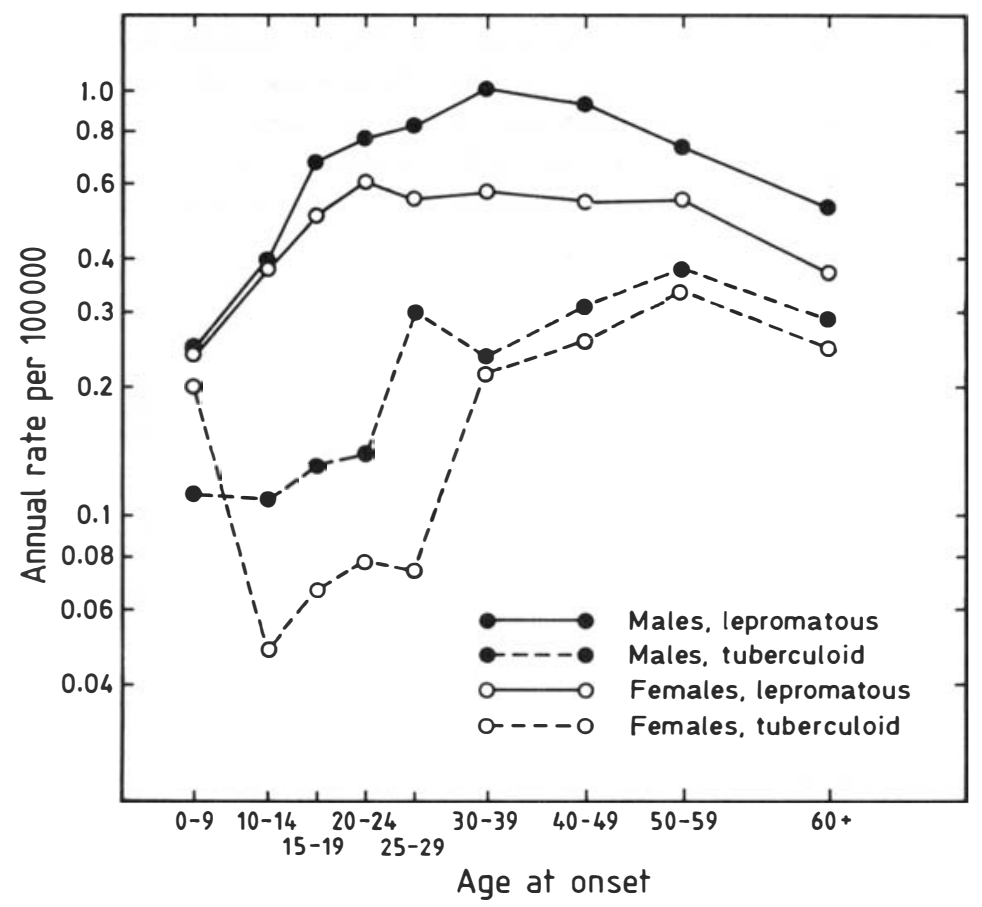

Figure 4. Average age, sex and type specific incidence rates of leprosy in continental Portugal, 1946-80.

observed in the districts with the highest rates, namely Coimbra and Leiria (Figure 1, Table 5). In these two districts the decline from 1946-50 to 1951-55 was relatively small suggesting a more stable level of approximately $8-10$ per 100,000 per year prior to 1945 .

The pattern in the distribution of new cases by age at onset changed considerably during the observation period implying an increasing age at onset (Figure 6). Thus the median age at onset changed from 30.0 years in $1946-50$ to 43.5 in $1976-80$, and the risk ratio based on age-specific incidence rates of $60+$ to $0-9$ years of age increased continuously from $1 \cdot 0$ in $1946-50$ to $39 \cdot 0$ in $1976-80$. 
Table 8. Proportion of all leprosy cases with age at onset less than 20 years by sex and average incidence rate in the district of residence of onset. Based on all cases in continental Portugal with year of onset 1946-80

\begin{tabular}{lcccc}
\hline & & \multicolumn{3}{c}{ Average incidence rate in district } \\
\cline { 3 - 5 } Sex & $\begin{array}{c}\text { Average } \\
(\%)\end{array}$ & $\begin{array}{c}-0 \cdot 74 \\
(\%)\end{array}$ & $\begin{array}{c}0 \cdot 75-3.0 \\
(\%)\end{array}$ & $\begin{array}{c}3 \cdot 1+ \\
(\%)\end{array}$ \\
\hline Male & $17 \cdot 8$ & 14.3 & 18.9 & $19 \cdot 7$ \\
Female & $18 \cdot 1$ & 14.4 & $19 \cdot 2$ & $19 \cdot 6$ \\
\hline Average & 18.95 & 14.3 & $19 \cdot 0$ & $19 \cdot 6$ \\
\hline
\end{tabular}

On the other hand in the analysis of birth cohorts this pattern was not found. If any trend at all, the results seemed to suggest rather the contrary: onset in consecutive birth cohorts tended to shift over to younger age groups (Figure 7).

Concomittantly, sex ratio increased from 1.2 in $1946-50$ to 1.7 in $1976-80$ (Figure 8). In lepromatous leprosy the sex ratio increased from 1.2 to 1.8 and in tuberculoid from $1 \cdot 1$ to $1 \cdot 4$.

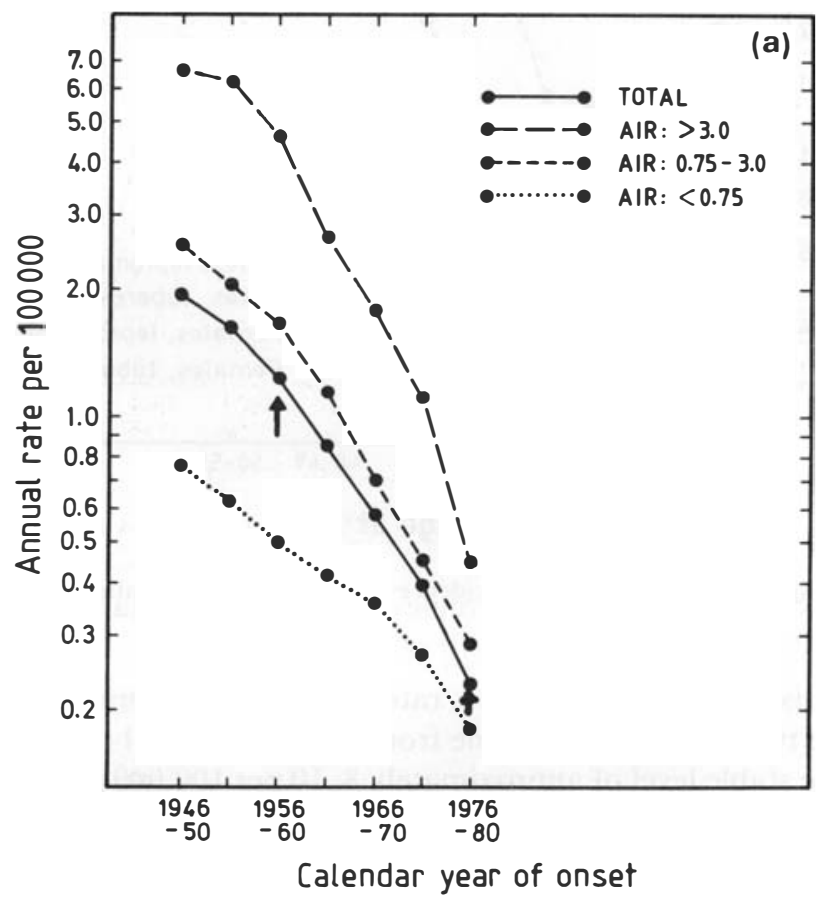

Figure 5. (a) Incidence rates of leprosy in continental Portugal, 1946-80, in districts grouped according to average incidence rates. Crude rates by year of onset. 


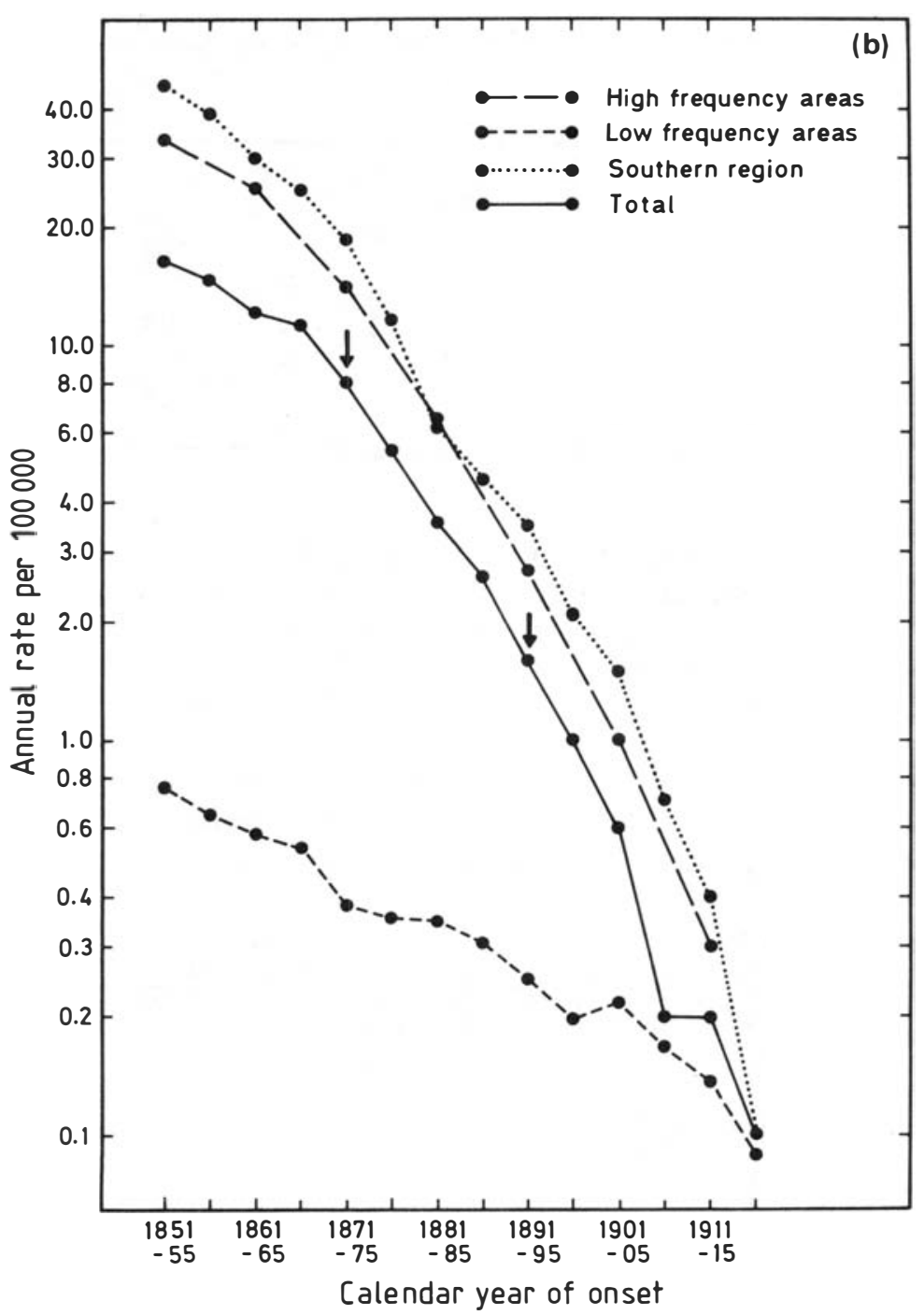

Figure 5. (b) Incidence rates of leprosy in Norway, 1851-1920 in various areas. Crude rates by year of onset ${ }^{1}$.

Type index decreased from $68 \cdot 0 \%$ in males and $64 \cdot 6 \%$ in females in $1946-50$ to $43 \cdot 1 \%$ and $34.7 \%$ respectively in 1976-80 (Figure 9).

\section{Discussion}

Problems of case ascertainment will relate to any collection of data used as a basis for epidemiological analyses, and particularly to data on a disease so closely associated with social stigma as leprosy. On the other hand, in a chronic disease for which a complete cure is obtainable, necessitating long periods of individual follow-up, medical records are essential. These conditions have formed the basis for epidemiological research in some countries. ${ }^{1,4-11}$ In Portugal a special administration was established devoted to leprosy 


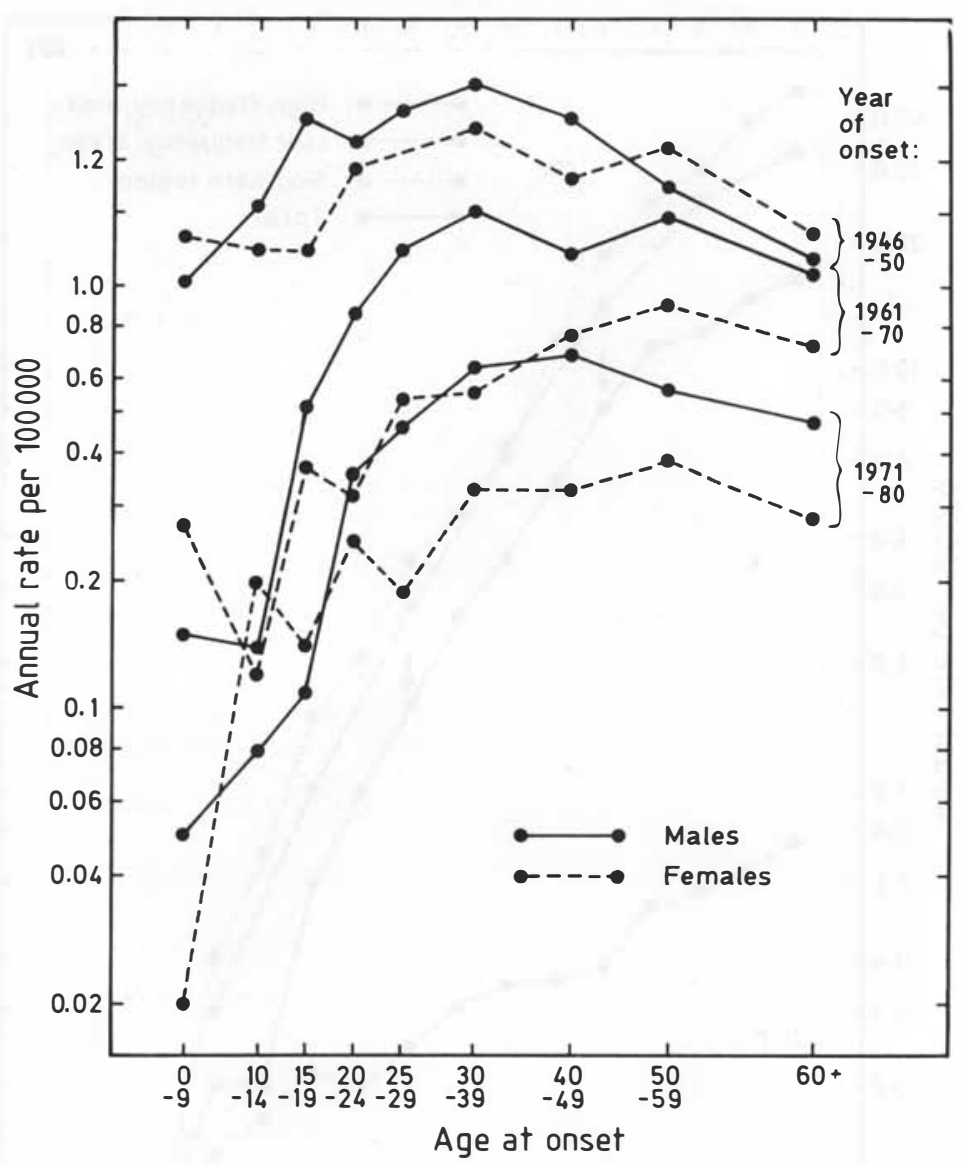

Figure 6. Incidence rates of leprosy in continental Portugal, 1946-80. Age-and sex-specific rates by year of onset.

control, ${ }^{2}$ and solely due to its organization there is no reason to believe that the data at hand are less reliable than those obtained in the other schemes. Furthermore, compared with the Norwegian Leprosy Registry, ${ }^{1}$ the delay in registration between onset and notification was somewhat shorter in Portugal $(0 \cdot 8-1 \cdot 8$ years against $1 \cdot 2-2 \cdot 0$ years in Norway (Table 3), while the data were slightly less complete (Table 4).

As in most other areas under epidemiological surveillance, the occurrence of the disease in Portugal varied considerably from district to district. First of all, leprosy was far more common in rural than urban areas, which apparently represents a general phenomenon in leprosy ${ }^{1,14-18}$ and supports the recommendation to search for and identify possible specific environmental factors. ${ }^{16}$ No negative association was found with urban development in terms of rapid population growth, so this aspect of urban dwelling apparently plays no role in the protection against leprosy.

Next, the association with rainfall observed in Norway ${ }^{1}$ was also found in Portugal, interacting with proximity to the sea, and implying a high incidence in coastal municipalities with a high annual rainfall. Since leprosy elsewhere is found also in a very 


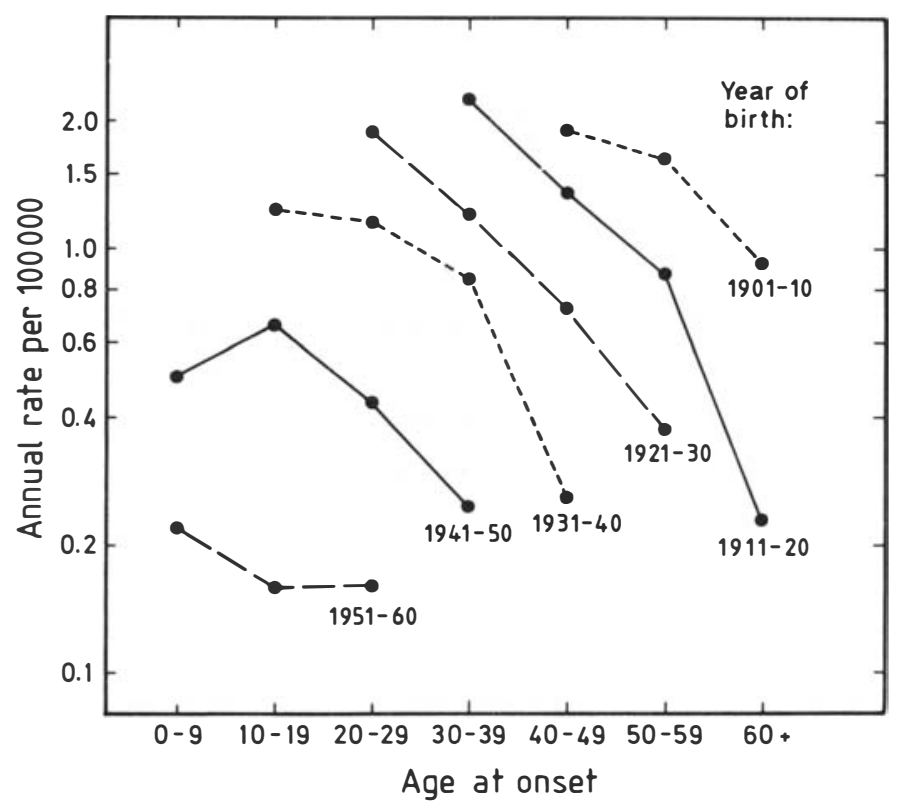

Figure 7. Incidence rates of leprosy in continental Portugal, 1946-80. Age-specific rates by year of birth.

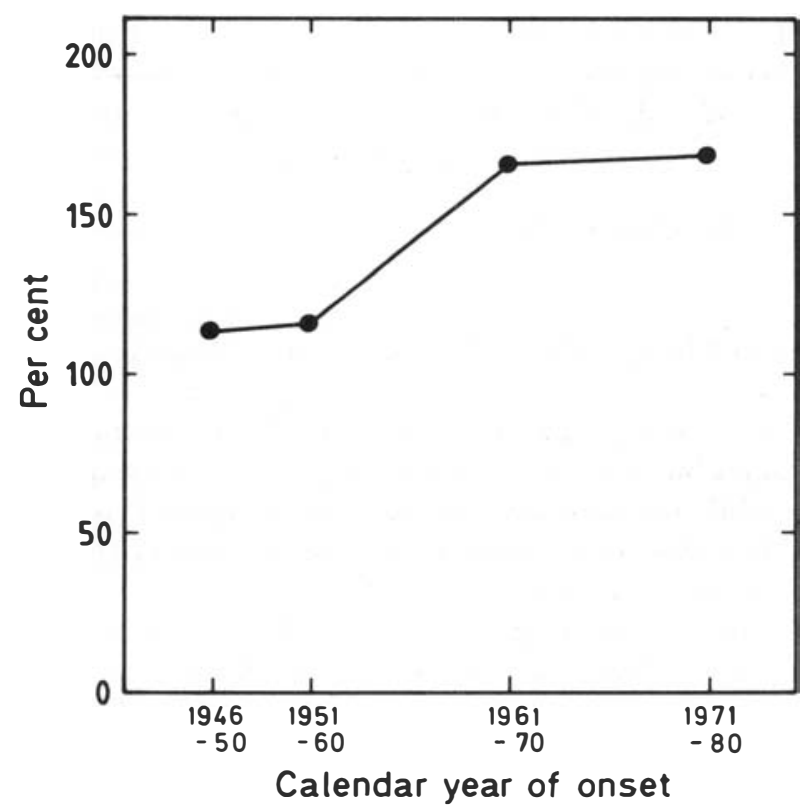

Figure 8. Sex ratio of leprosy in continental Portugal, 1946-1980, by year of onset. Ratio based on sex-specific incidence rates. 


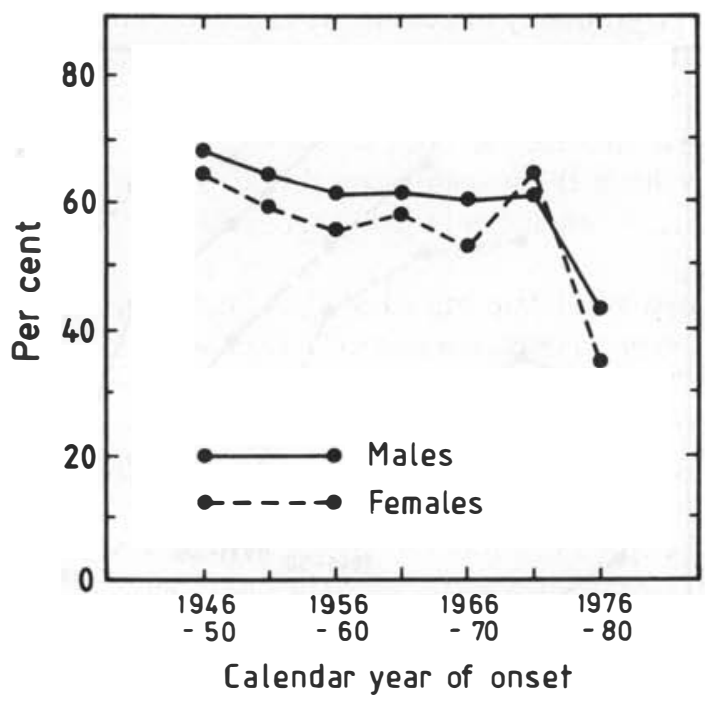

Figure 9. Type index of leprosy in continental Portugal, 1946-80, by sex and year of onset.

dry environment, high air humidity does certainly not represent a necessary aetiological factor. However possible sources of environmental Mycobacterium leprae $e^{17,19,20}$ may be influenced by climatic variables, of which air humidity seems relevant also from an ecological point of view. The issue will not be further discussed here but needs clarification in future studies.

As in most other areas under epidemiological surveillance, leprosy was more frequent in males than in females. Peak incidence rates in males were observed at 25-29 years of age against 50-59 years in females (Figure 3). If females are mainly infected within the household, i.e. early in life, the opposite would have been anticipated which has also been observed in other studies. ${ }^{13}$ Whether the finding in Portugal is caused by a longer period of diagnostic delay in females will not be further pursued. Moreover, age at onset was higher in tuberculoid than in lepromatous leprosy, particularly in women (Figure 4). A longer incubation period in lepromatous leprosy would suggest the opposite finding. However, a delay in casefinding in less conspicuous (i.e. tuberculoid) females might explain this result as well.

As in Norway the Portuguese data covered a period of continuous decline. No sources are available to assess whether the decline started from a plateau in the 1930-40s or whether the rates were far higher at the beginning of the century. Still, the decline observed was considerable, representing a ratio of risks from 1946 to 1980 of 8.2. The annual relative decline, ${ }^{13}$ calculated as

$$
n \sqrt{ } i_{o} / i_{n}
$$

where $n$ denotes number of years (here, 35) and $i_{o}$ and $i_{n}$ the incidence rates of the first and the last 5 -year periods (here, 1.96 and 0.24 per 100,000 per year respectively), was 1.06 which is close to the declines observed in Norway and recently in Venezuela. ${ }^{13}$ Also, for this reason, the data seem comparable with those of studies already published on declining incidence rates. ${ }^{1,4-11}$

In many ways the decline bore a striking resemblance to the development observed in 
Norway. Not only did a gradually increasing decline occur, but the slope of the incidence curve in Norway, 1871-95 (Figure 5(b)) was identical with the one in Portugal, observed in 1956-80 (Figure 5(a)). Admittedly some factors (which might or might not be identical), have caused the decline both in Norway and Portugal, but certainly the question arises-why have these factors produced the same slope of decline in two countries so far from each other, not only in space and time, but also in terms of leprosy control strategy?

Most likely the slope is not determined by these factors. Rather the pattern of decline probably represents a general phenomenon in leprosy which occurs af ter the spread of the infection has ceased. Thus the incidence curve may, to a large extent, be interpreted as reflecting the right part of the distribution curve of the incubation period in leprosy. The slope of the incidence curve reflects the diminishing number of cases observed towards the right tail of the distribution curve, and is apparently not determined by the factors causing the interruption of the spread of the infection. Furthermore, the form of the incidence curve on a logarithmic scale is consistent with the principle suggested by Sartwell that the frequency curve of incubation time takes the form of a logarithmic normal curve. ${ }^{21}$

This interpretation sheds light on a phenomenon observed in Norway. ${ }^{1}$ During a period of high incidence (i.e. still spread of the infection), isolation of infectious cases was strongly associated with the subsequent decline of the incidence rate, while towards the end of the epidemic (i.e. the part of the incidence curve determined by the incubation period), this association gradually vanished; leprosy disappeared regardless of the degree of isolation.

Towards theend of the epidemic in Portugal the age specific incidence rates showed, to an increasing extent, that new cases represented patients of higher age (Figure 6). This trend might be caused by two mechanisms. ${ }^{22}$ Either a postponement of infection to a higher age has led to a higher age at onset. Alternatively it might be caused by the possibility already mentioned that the late patients had longer incubation periods. Infected when incidence rates were higher they would tend to be more frequent than the younger patients with the same period of onset, the latter infected when incidence rates were lower and thus less frequent. A cohort analysis (Figure 7) did not show an increasing age at onset in consecutive birth cohorts, and thus ruled out the possibility of a postponement of infection to higher age groups. This finding also supports the notion that the slopes of the incidence curves are influenced by cases with increasingly long incubation periods. Similar trends are observed in some countries in which data for such analyses are available. ${ }^{1,4,9,13,22}$

However, this does not rule out a mechanism of a postponement of infection in other situations. The mechanism is most likely the cause of the relatively low proportion of young patients notified in low incidence areas (Table 7), where infection in the community at large is supposed to play a relatively more important role (vide infra).

Sex ratio was always above 100 and increased during the observation period, and more so in lepromatous than in tuberculoid leprosy. Similar trends have been observed also in other countries. ${ }^{21}$ Apart from a registration bias, women leading more secluded or protected lives within the household (i.e. 'protected' against notification), the higher rates in males may reflect that males, in addition to the risk of household infection, which is equal in the two genders, also bear a risk of being infected in the community at large. Risk of infection in the community is higher in the gender that has the most extensive extra household contacts, i.e. usually males. 
The secular trend of an increasing sex ratio is consistent with the fact that during declining incidence rates, the risk of getting infected in the household declines due to a declining number of leprosy infected households. The risk of infection in the community, on the other hand, remains as long as infectious patients exist within the area. Thus the relative importance of infection in the community increases, which is consistent with the increasing sex ratio observed. Alternatively a shorter incubation period in females could effect the same pattern. However, a high sex ratio observed in low incidence areas (Table 6) supports the former interpretation.

The interpretation of the data on type index may involve far more uncertainty. Thus it may be speculated that a more pronounced secular increase of sex ratio in lepromatous than in tuberculoid leprosy may be related to the assumption that only persons with a high susceptibility are infected (and subsequently taken ill) in the community at large (namely, males), and these persons develop lepromatous leprosy due to their low resistance. This may also explain why type index in general is higher in males than in females. On the other hand the secular decline of type index observed in both genders in Portugal (Figure 9) is not consistent with an increasing proportion of cases with long incubation periods, provided lepromatous cases have the longest incubation periods, nor with an increasing relative importance of infection in the community at large. In China, ${ }^{4} \mathrm{Japan}^{6,7}$ and Venezuela ${ }^{10}$ an increasing type index has been observed towards the end of the epidemic, while in India ${ }^{5}$ the opposite trend has been observed (i.e. the same as in Portugal). These conflicting observations may be due to two different lines of event: on one hand, an increasing resistance in the population against leprosy produces both a fall in incidence and a lower proportion of lepromatous cases, while, on the other hand, longer incubation periods and greater importance of infection in the community at large (compared to infection in the household) tend to give a higher proportion of lepromatous cases. Thus, in Norway, an increasing type index was observed in males against a decreasing type index in females. ${ }^{1}$

Also other difficulties arise in the interpretation of the data. Thus, the age distribution of new cases indicates a higher age at onset in females than in males (Figure 3), which is inconsistent with the hypothesis of females leading more secluded lives and thus not being infected in the community at large; however, this pattern changed by time (Figure 6). Later, a shift towards higher age groups was particularly observed in males consistent with an increasing importance of infection in the community at large.

On the other hand the age distribution of cases suggests a higher age at onset in tuberculoid than in lepromatous leprosy (Figure 4), inconsistent with a longer incubation period in lepromatous leprosy. In the several epidemiological schemes now under way the clarification of such issues represents important aims necessary to obtain a more comprehensive understanding of the epidemiology of leprosy.

\section{Acknowledgments}

Acknowledgment is made of the financial support provided by the Damien Foundation, Belgium to conduct this study. The authors are indebted to the Health Directorate of Portugal for fruitful co-operation and to the personnel in Portugal involved in the registration of data and the running of the register. The authors are also indebted to The 
Section of Medical Informatics and Statistics, University of Bergen, Norway, for valuable assistance.

\section{References}

1 Irgens LM. Leprosy in Norway, an epidemiological study based on a national patient registry. Lepr Rev, 1980; 51 (Suppl. 1): 1-130.

${ }^{2}$ Melo Caeiro F, Teixeira J, Pereira F. Hansen's Disease in Portugal-the Situation in 1984. O Medico, 1985; 1742: $825-83$.

3 Servico Meteorologico Nacional. Normais Climatologicas: 1931-1960. Lisboa: Edicao preliminar, 1974.

4 Li HY, Pan YL, Wang Y. Leprosy control in Shandong Province, China, 1955-1983; some epidemiological features. Int J Lepr, 1985; 53: 79-85.

5 Vanderverken M, Lechat MF, Misson CB, Vellut C, Antony VV. Age-, sex-, type-specific incidence rates in leprosy. Observation on 45,000 leprosy patients detected in Polambakkam, South India over a 27-year period. Abstr. in Int $J$ Lepr, 1985; 53: 740.

6 Ito T. The epidemiological situation in South East Asia. Lepr Rev, 1981; 52 (Suppl.): 43-51.

7 Saikawa K. The epidemiological phenomenon on decreasing tendency of leprosy disease. Jpn J Lepr, 1981; 50: $99-104$.

${ }^{8}$ Davey T. Decline of leprosy in a group of Nigerian villages between 1941 and 1956. Int J Le pr, 1957; 25: 32944.

9 Feldman R, Sturdivant M. Leprosy in Louisiana, 1855-1970. An epidemiologic study of long-term trends. Am J Epidemiol, 1975; 102: 303-10.

10 Zuniga M, Castellazzi Z. 30 anos de evolucion de la endemia de la le pra en Venezuela (1949-1979). Caracas: Cepialet, 1982.

11 Christian M. The epidemiological situation of leprosy in India. Lepr Rev, 1981; 52: 35-42.

12 Statistical Package for the Social Sciences (SPSSX), 2nd ed. Chicago: McGraw Hill, 1986.

13 Irgens LM, Skjærven R. Secular trends in age at onset, sex ratio and type index in leprosy observed during declining incidence rates. Am J Epidemiol, 1985; 122: 695+705.

14 Leiker DL. Some aspects of the epidemiology of leprosy: on the relationship of leprosy and other mycobacterial diseases. Int J Lepr, 1971; 39: 610-15.

15 Guinto RS. Epidemiology of leprosy; current views, concepts and problems. In $A$ window on leprosy, Chatterjee BR, (ed). Delhi: Ghandi Memorial Leprosy Foundation, 1978: 36-53.

${ }^{16}$ WHO Expert Committee on Leprosy. Sixth report. Technical Report Series No. 768, 1988.

17 Blake LA, West BC, Lary CH, Todd JR. Environmental nonhuman sources of leprosy. Review's of infectious diseases, 1987; 9 no. 3: 562-77.

18 Fine PEM. Leprosy: the epidemiology of a slow bacterium. Epidemiological Review's, 1982; 4: 161-88.

19 Kazda J, Ganapati R, Revankar C, Buchanan TM, Young DB, Irgens LM. Isolation of environment-derived Mycobacterium le prae from soil in Bombay. Lepr Rev, 1986; 57 (Suppl. 3): 201-8.

20 Irgens LM, Kazda J, Müller K, Eide GE. Conditions relevant to the occurrence of acid-fast bacilli in sphagnum vegetation. Acta Path Microbiol Scand Sect B, 1981; 89:41-7.

${ }_{21}$ Sartwell PE. The distribution of incubation periods of infectious disease. Am J Hyg, 1950; 51: 310-18.

22 Irgens LM. Secular trends in leprosy: increase in age at onset associated with declining rates and long incubation periods. Int $J$ Lepr, 1985; 53: 610-17. 